\title{
License Plate Detection and Recognition using OCR based on Morphological Operation
}

\author{
Thida Win \\ Yangon,Myanmar
}

\author{
Dr. Hnin Ei Latt \\ Yangon, Myanmar
}

\author{
Dr. Yin Mon Swe \\ Yangon, Myanmar
}

\begin{abstract}
License Plate Recognition has been intensively studied in many countries. Due to the different types of number plates being used, the requirements of an automatic number plate recognition system is different for each country. In this paper, a number plate localization and recognition system for vehicles in Myanmar is proposed. This system is developed based on digital images and can be easily applied to toll gate, car parking systems for the use of documenting access of parking services, secure usage of parking houses and also to prevent car theft issues. The proposed algorithm is based on colour based detection and k-Nearest Neighbour(k-NN) with area criteria tests for number plate localization. The next feature is the Euler Number, which is the same as the number of holes in a binary image. Segmentation of the plate characters was achieved by the application of bwboundaries. Removing noise using morphological operation. The character recognition was accomplished with the aid of optical characters recognition(OCR)by the process of Pattern matching. A comparative analysis on the success rate of the proposed system showed overall better success rate of $96.8 \%$ by using OCR.
\end{abstract}

Keywords: License plate recognition, colour based detection, morphology operation, k-NN, optical character recognition.

\section{INTRODUCTION}

Each vehicle has a unique identification number which is called license plate (LP). The license plate has a rectangular shape and contain two parts. Upper part has regional division and the lower part has a group of numbers and letters. Because the growth number of vehicles, which leads to many vehicles related issues, license plate recognition [1]became a very important .In the current information technology era, the use of automations and intelligent systems is becoming more and more widespread. License plate recognition has turned out to be an important research issue. License plate recognition has many applications in toll gate, car parking systems for the use of documenting access of parking services, secure usage of parking houses and also prevent car theft issues and so on.[3]

The system of license plate in Myanmar is based on the residency area in which the vehicle is registered and on the registration order. Some countries have many versions on the form of license plate, for example Tunis, China, Libya, and India. Besides the form, Myanmar also applies colour to the system of license plate, i.e. private vehicle (black), taxi/bus vehicle (red), vehicle owned by monk and/or monastery (yellow), touring vehicle by (blue),and vehicle owned by Ambassador (white). This research is aimed to create a license plate recognition system. This system later can be used as there ference to identify vehicles. The challenge in the process of license plate identification is how to detect and recognize the license plate of vehicles in different type,especially red colour and black colour vehicle plate in Myanmar.

Some previous research in recognition of Indonesian license plate have been carried out. A research employing Fourier transform method and hidden markov model obtained an accuracy of $84.38 \%$. Another research resulted accuracy value of $85 \%$ on the character recognition and of $97 \%$ on the process of detecting the location of license plate. This research uses contour in detecting the location. The process continues by doing figure segmentation utilizing interconnected figure segmentation, and is ended in the process of classification by using static classification technique by which the segmented figure is translated into the character of ASCII. However, a problem aroused when a character cannot be recognized because of its different shape or italic shape. A high degree of accuracy, that is $96 \%$, is gained in the other previous research.[3]

This research implemented a method of colour based detection to extract feature and k- nearest neighbour(k$\mathrm{NN}$ ) as the classification method optical character recognition(OCR) was used to recognize license plate.

\section{RELATED WORKS}

The performance of license plate recognition, which use colour based detection to detect and extract location of the license. The license plate properties extraction is obtained and k-Nearest Neighbour(k-NN) based classification. Additionally Hole(Euler Number) was used for filtering. And then, morphological operation was used for removing noise. And Segmenting the each character used bwboundaries function. Finally,opticalcharacter recognition(OCR) was used to recognize the license plate.

In recent years, some developed countries created license plate recognition systems for many applications of their traffic requirements. In regard to plate localization, many techniques have been used in connected domain analysis, mathematical morphology colour model, fuzzy set theory, and statistical classifications. For license plate recognition, mainly the recognition techniques can be divided in to two main categories. The template based method and the supervised learning method as mention in . The template based method usually used in character recognition by comparing each character with stored characters to find 
the exact matching or the closest character. The matching technique used is normalized correlation method to indicate how well the chosen character matches with the stored character. This method is sensitive to the noise disturbance, and image orientation. While the supervised-based method has common classifiers that have been used for character recognition, Mark net and Bayes net have been used as mention in, which are Neural Network (NN) as mention in, and support vector machine (SVM) as mention in. Because of the rapid development in digital signal processing and digital image processing, many systems implemented on an embedded digital system to process video stream, such as in .The system consist of modules to detect and recognize characters. AdaBoost technique used to detect license plate, and cascade framework used for license plate recognition, Ami the most recent two decades many research endeavors have been spent to build up the license plate acknowledgment system., some of the distributed work is exhibited, the emphasis will be on the technique utilized for executing those LPR frameworks.[2]

$$
\text { where } \begin{aligned}
I_{b w}(i, j) & =\text { matrix value of RGB } \\
i & =\text { row of matrix } \\
j & =\text { column of matrix }
\end{aligned}
$$

\section{B. $k$ - nearest neighbours}

k-Nearest neighbours algorithm is a method for classifying objects based on closest training examples in the feature space. k-nearest neighbours algorithm is among the simplest of all machine learning algorithm. Training process for this algorithm only consists of string feature vectors and labels of the training images. In the classification process, the unlabeled query point is simply assigned to the label of its knearest neighbours. Typically the object is classified based on the labels of its $k$ nearest neighbours by majority vote. If $k=1$, the object is simply classified as the class of the object nearest $\mathrm{t}$ it. When there are only two classes, $k$ must be an odd integer. However, there can still be tie when $k$ is an odd integer when performing multiclass classification. After we convert each image to a vector of fixed-length with real numbers, we used the most common distancefunction for k-NN which is Euclidean distance between the points $\mathrm{x}$ and $\mathrm{u}$ is

$$
\begin{aligned}
\boldsymbol{d}(\boldsymbol{x}, \boldsymbol{u})= & \sqrt{\sum_{i=1}^{n}\left(\boldsymbol{x}_{\boldsymbol{i}}-\boldsymbol{u}_{i}\right)^{2}} \\
\text { where, } \mathrm{d}(\mathrm{x}, \mathrm{u}) & =\text { distance from the sample vector } \\
\mathrm{x} & =\text { feature of training image } \\
\mathrm{u} & =\text { feature of test image }
\end{aligned}
$$

\section{Hole (Euler Number)}

The Euler number, which is the same as the number of holes in a binary image. It is calculated by morphological function. The ratios of holes area to the entire area is another feature, which can be determined when the system calculates the Euler number. Other sample features, such as pressing ration (ratio of object pixels to all pixels), are useful for accurate recognition.

\section{BACKGROUND THEORY}

The performance of license plate recognition, which use colour based detection to detect and extract location of the license. The license plate properties extraction is obtained and k-Nearest Neighbour(k-NN) based classification. Additionally Hole(Euler Number) was used for filtering. And then, morphological operation was used for removing noise. And Segmenting the each character used bwboundaries function. Finally, optical character recognition(OCR) was used to recognize the license plate.

\section{A. colour based detection}

Because colour information is used to detect the location of a license plate, converting the whole image into gray level, into black and white level, or binary level is not necessary. Detecting an object within its image has always been difficult in image processing. Colour features to localize a license plate.

$$
I_{b w}(i, j)= \begin{cases}1 & \text { ifdesiredRGB } \\ 0 & \text { otherwise }\end{cases}
$$

\section{Euler Number=1- Number of holes in that object}

\section{Morphological operation}

Mathematical morphology analyses images by using operator developed using set theory. It was originally developed for binary image was extended to include grey-level data. The word morphological concerns shapes. In this way morphological operators define local transformations that change pixel values that are represented as sets, he ways in which pixel values changed is formalized by the definition of the hit or miss transformation. There were many operators such as dilation, erosion, reconstruction-based operator and other operations. bwareaopen function was used to remove noise.

bw2=bwareaopen(bw,p)--------------------------------3

Here, removes from a binary image all connected objects that have fewer the p pixels, producing another binary image bw2. This operation is known as an area opening.

\section{Segmentation}

Segmentation using bwboundaries.Bwboundaries is a cell array in which each element is the boundary of an object in the binary image $b w$. The clockwise boundary of each object is computed by the boundary function. By default the boundaries are computed using 8connectivity.

$[\mathrm{B}, \mathrm{L}]=$ bwboundaries(bw,'noholes');-------------------4 where, $[\mathrm{B}, \mathrm{L}]=$ returns a label matrix $\mathrm{L}$

bw= binary image

noholes $=$ search only for object (parent and child)

\section{E. Optical Character Recognition(OCR)}


Recognizing text in images is useful in many computer vision applications such as image search, document analysis, and robot navigation. The OCR function provides an easy way to add text recognition functionality to a wide range of application. The OCR functions returns the recognized text, the recognition confidence, and the location of the text in the original image. OCR performs best when the text is located on a uniform like a document. OCR is a field of research in pattern recognition, artificial intelligence and computer vision. OCR can recognize text such as, "Block" or "Line" or "Word". And OCR can declared language such as "English" or "Japanese".

There are two basic types of core OCR algorithm, matrix matching and feature extraction. Matrix matching involves comparing an image to stored glyph on a pixelby-pixel basis; it is known as "pattern matching", "pattern recognition', or "image correlation". Feature extraction decomposes glyphs into "features" like lines, closed loops, line direction, and line intersections.[4]

T=ocr(Ibw,"TextLayout","Block","Language","Engli sh");

where, $\mathrm{T}=$ return value

Ibw= binary image

\section{EXPERIMENTAL RESUlTS}

A. Design of the proposed system

The proposed system design is as shown in figure 1 .

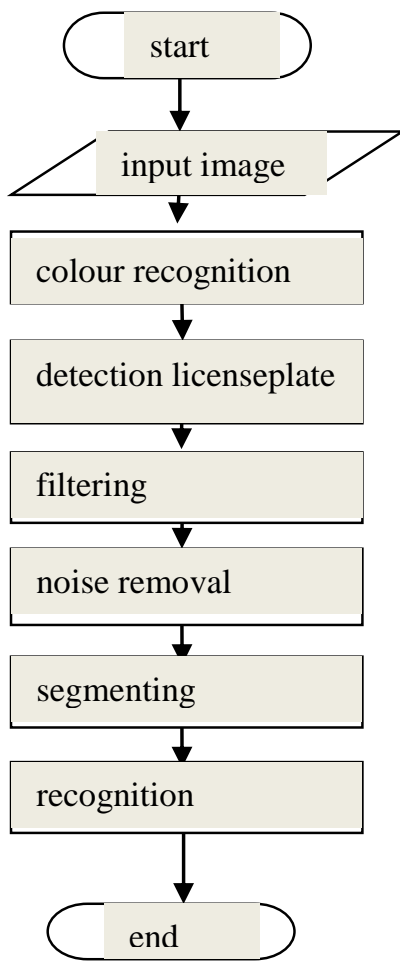

Fig.1Flow chart of the proposed system
The proposed system consists two parts in implementation. Firstly, the extraction and localization of the license plate. Then, recognition of license plate was perform.This system inthe processof license plate identification is how to detect and recognize the license plate of vehicles in different form and type especially red colour and black colour vehicle plate in Myanmar.

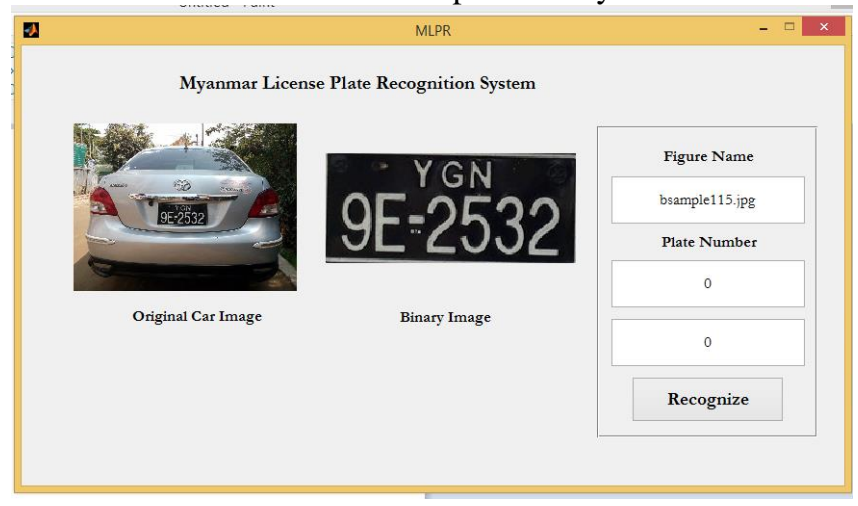

Fig. 2Extraction and localization of saloon type black colour

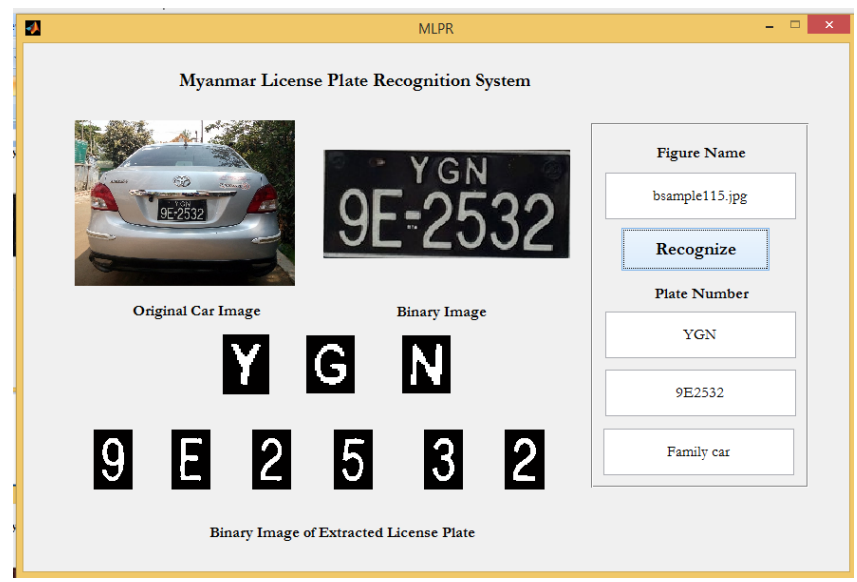

Fig. 3 Recognition of saloon type black colour

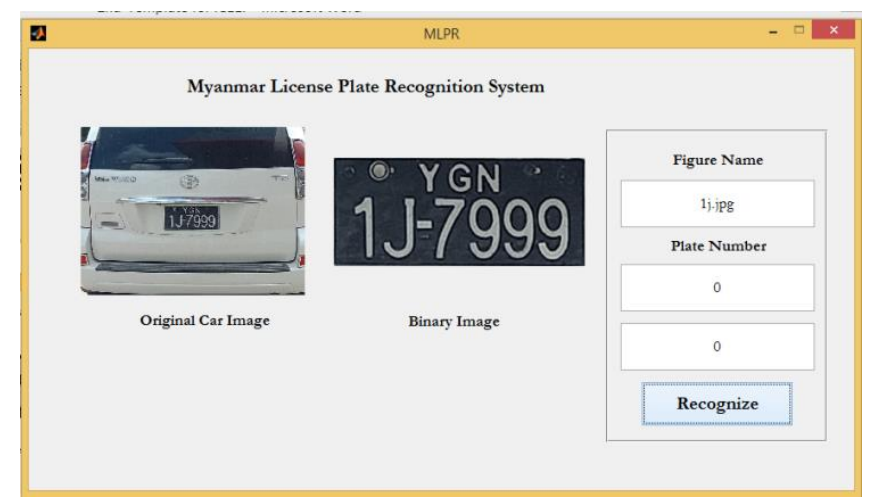

Fig. 4Extraction and localization of surf type black colour

\section{B. Implementation of the proposed system}




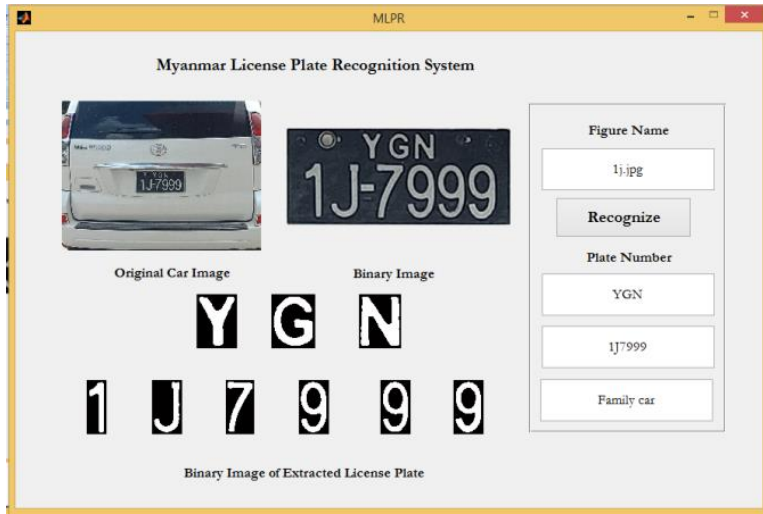

Fig. 5Recognition of highsurf black colour

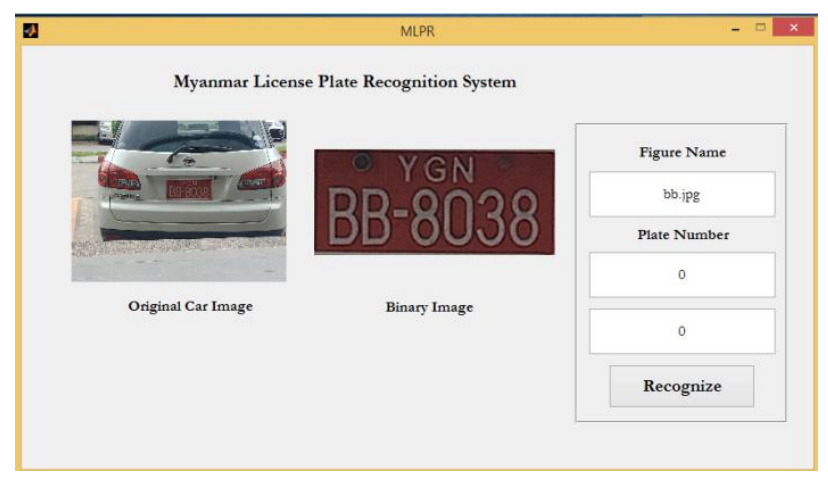

Fig. 6Extraction and localization of taxi

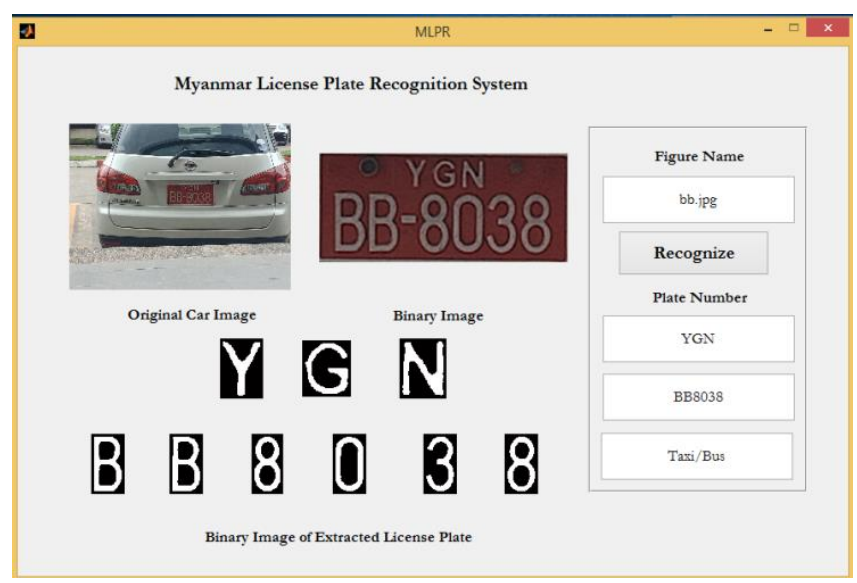

Fig. 7Recognition taxi

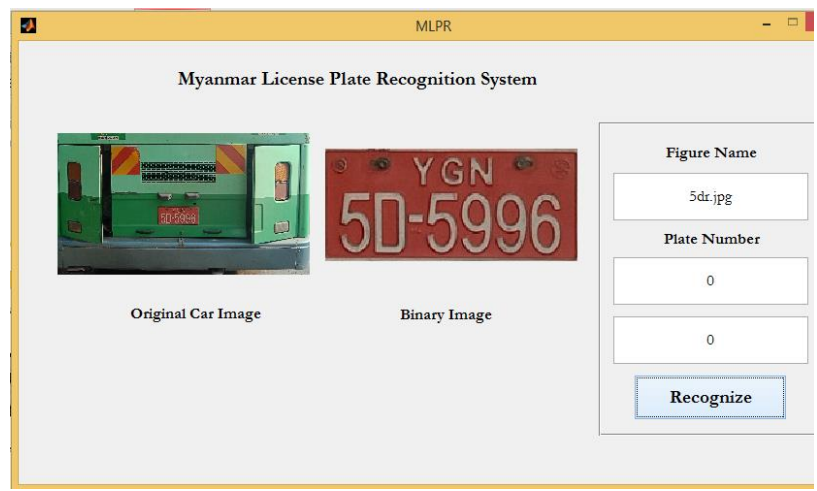

Fig. 8Extraction and localization of bus

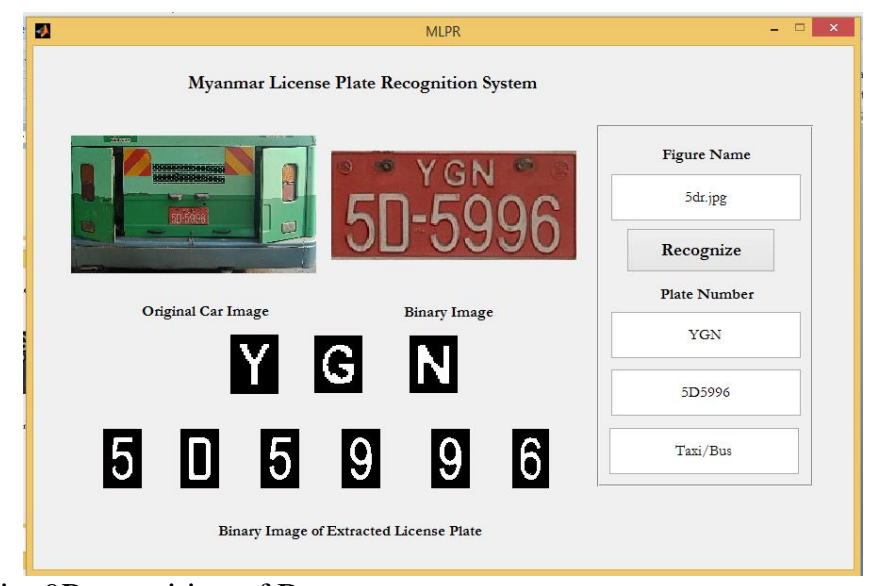

Fig. 9Recognition of Bus

TABLE I

EXPERIMENTAL RESULTS

\begin{tabular}{|l|l|l|l|}
\hline \multirow{2}{*}{ NO } & \multicolumn{3}{|c|}{ EXPERIMENTAL RESULT } \\
\cline { 2 - 4 } & Car Type & Color & percentage \\
\hline 1 & Saloon type & black & 75 \\
\hline 2 & Surf & black & 65 \\
\hline 3 & Taxi & red & 72 \\
\hline 4 & Bus & red & 35 \\
\hline
\end{tabular}

The above table show the percentage of result test. The training data set is 26 alpha bats and 10 digits . Total of test data is based on 100 images.

\section{CONCLusions}

This paper presents a proposed technique to detect and recognize the Myanmar license plate based on $\mathrm{kNN}$ algorithm and OCR. The image for the license plate first pre-processed through several steps; to specify the plate location in the image then cutting the plat part from the image. Then, according to the myanmar license plate features, the proposed algorithm separates the characters and the numbers in the plate, and recognize each character. Next implementation is to handle more various complex conditions such as blurring, low resolution, character noise by enhancing the robustness and selection more character features to increase the character recognition in such conditions. The weak point of OCR algorithm is can recognize "O" as "Q". It can also use ".jpg" and ".png" format. The proposed system should perpendicular with camera.

\section{ACKNOWLEDGMENT}

The author wishes to express her deeply thankful to Dr.Kay ThiLwin, Rector Technological University (Hmawbi), for her invaluable attitude, suggestions and encouragement for the completion of this paper. The author is sincerely grateful her chairman Dr.SoemoeOo, Professor and Head, department of Information Technology, Technological University (Hmawbi). The author is also especially indebted to her supervisor, Dr.HninEiLatt, Lecturer, Technological University 
(Hmawbi) for her imagination, enthusiasm, expertise and technical knowledge in diversified areas.

\section{REFERENCES}

[1] abdulhadimohammedSaif Ali AbdAlradhaAlsaidi Omar HashimYahya" Plate Detection and Recognition of Iraqi License Plate Using KNN Algorithm, 9449-460.

[2] Amir HosseinAshtari, Graduate Student Member; IEEE, Md. Jan Nordin, and MahmoodFathy (2014)."An Iranian License Plate Recognition System Based on Color Features".journal of IEEE Transactions on Intelligence Transportation Systems, 15(4), 1690-1705.

[3]P.ANISHIYA 1, PROF. S. MARY JOANS 2(1022)."Number Plate Recognition for Indian Cars Using Morphological Dilation and Erosion with the Aid Of Ocrs", International Conference on Information and Network Technology,,vol 4,(115-119).

[4] Fitri Damayanti1, Sri Herawati2, Imamah3, FifinAyu M4, AeriRachmad*5 (2019 April)"Indonesian license plate recognition based on area feature extraction"."TELKOMNIKA", Vol.17, No.2,620-627.

[5] M. Sarfraz, M.J. Ahmed, S.A. Ghazi (2003), "Saudi Arabian license plate recognition system, GeometricModeling and Graphics Proceedings".International Conference on, IEEE2003, .36-41.

[6] M. Kumar (2009), "A Real-Time Vehicle License Plate Recognition (LPR) System", THAPAR UNIVRSITY PATIALA.

[7] W. Al Faqheri, S. Mashohor,(2009) "A real-time Malaysian automatic license plate recognition (M-ALPR) using hybrid fuzzy", IJCSNS International Journal of Computer Science and Network Security, vol. 9, 333-340.

[8] Z. Wang, S. Li (2010)."Research and implement for vehicle license plate recognition based on improved BP network", 2010 International Conference on Computer and Communication Technologies in Agriculture Engineering, IEEE2010. 101-104. 